\title{
The Effect of Psychological Anxiety Factors Felt by Dental Hygienists in Clinical Practice on Job Performance
}

\author{
Hyun Suk Kang ${ }^{1}$, Sun Ae Kwon ${ }^{1}$, Min Jeong Kim ${ }^{1}$, Yun Yeong Kim ${ }^{1}$, Moon Yong Choi ${ }^{1}$, Young Eun Lee ${ }^{1}$ \\ ${ }^{1}$ Department of Dental Hygiene, Daegu Health College, Daegu, Republic of Korea
}

Objectives: For dental hygienists to have long-term self-satisfaction in the clinical practice and to perform high-quality health work, it is an essential factor that helps improve the oral health of patients. On the other hand, although dental hygienists are professional workers, their job lifespan is reported to be as low as 10 years or less. Psychological anxiety factors felt in clinical practice can be cited as the cause. Therefore, in this study, we tried to find out the psychological anxiety factors that dental hygienists feel in the field, and to investigate the effect of those anxiety factors on their job ability.

Methods: This study conducted an online survey on May 2021, targeting 128 dental hygienists currently working in dental clinics, dental hospitals, university hospitals, and general hospitals nationwide who agreed to participate in the survey were included in the study. A structured self-report questionnaire consisting of a total of 7 items and 56 questions was used.

Results: As the most difficult point at the current workplace, work stress was the highest at $44.5 \%$. As for the highest portion of psychological anxiety felt at the hospital, $43.0 \%$ were due to one's ability, and $33.6 \%$ were due to communication with colleagues and patients. These psychological factors were found to have a significant effect on job ability.

Conclusions: In conclusion, it is considered that actively seeking ways to resolve psychological anxiety factors that can affect dental hygienists' job ability in clinical practice is an essential way to improve the job satisfaction of dental hygienists and to lower the turnover rate.

Keywords: Psychological anxiety factors, Job ability, Dental hygienists

Copyright (C) 2021. Korean Academy of Preventive Dentistry. All rights reserved.

This is an Open Access article distributed under the terms of the Creative Commons Attribution Non-Commercial License (http://creativecommons.org/licenses/ by-nc/4.0) which permits unrestricted non-commercial use, distribution, and reproduction in any medium, provided the original work is properly cited. 